\title{
Japanese physicians' attitudes towards enteral nutrition treatment for pediatric patients with Crohn's disease: a questionnaire survey
}

\author{
Takashi Ishige ${ }^{1}$, Takeshi Tomomasa ${ }^{2}$, Hitoshi Tajiri ${ }^{3}$, Atsushi Yoden ${ }^{4}$, on behalf of the Japanese Study \\ Group for Pediatric Crohn's Disease \\ ${ }^{1}$ Department of Pediatrics, Gunma University Graduate School of Medicine, Maebashi, ${ }^{2}$ PAL Children's Clinic, Isesaki, \\ ${ }^{3}$ Department of Pediatrics, Osaka General Medical Center, Osaka, ${ }^{4}$ Department of Pediatrics, Osaka Medical College, Takatsuki, Japan
}

\begin{abstract}
Background/Aims: Enteral nutrition (EN) is recommended for the treatment of pediatric Crohn's disease (CD) in Japan. However, the indications and treatment protocols for EN vary among hospitals. In the present study, we aimed to determine how EN was administered to pediatric patients and whether physicians followed treatment guidelines in their practices. Methods: Two types of questionnaires were administered to 32 physicians who were involved in the treatment of pediatric CD. The consensus questionnaire evaluated the physicians' attitudes towards EN, whereas the efficacy questionnaire collected data on patients with $\mathrm{CD}$, aged $<17$ years, who had undergone induction therapy between 2006 and 2011. Results: A total of 23 physicians responded to the questionnaires. The results of the consensus questionnaire indicated that $82 \%$ and $59 \%$ of study participants recommended EN treatment for all newly diagnosed pediatric patients with CD and all relapsed patients, respectively. Exclusive EN (EEN) and elemental formula were recommended by $84 \%$ and $85 \%$ of physicians, respectively. The efficacy questionnaire revealed that 57 of the 58 patients received EN. Elemental formula was used in 39 of 40 patients who were treated with EEN. Of these 40 patients, 27 were treated with EEN alone; of these, 22 (81\%) achieved remission without any other treatment. The mean duration of EEN was 15.9 days. Conclusions: EN is widely recommended by physicians treating pediatric CD in Japan. In contrast to Western countries, clinicians used elemental formula more often and with a shorter EEN treatment duration. (Intest Res 2017;15:345-351)
\end{abstract}

Key Words: Crohn disease; Food, formulated; Enteral nutrition; Child; Surveys and questionnaires

\section{INTRODUCTION}

Crohn's disease (CD) is a chronic recurring condition that can affect any part of the gastrointestinal tract. Approximately $10 \%$ of patients newly diagnosed with IBD in Japan are children and adolescents aged $<17$ years. ${ }^{1}$ Growth and nutrition are vital considerations in the management of ado-

Received September 17, 2016. Revised November 22, 2016. Accepted December 1, 2016. Published online March 31, 2017

Correspondence to Takashi Ishige, Department of Pediatrics, Gunma University Graduate School of Medicine, 3-39-22 Showa-machi, Maebashi 371-8511, Japan. Tel: +81-27-2208205, Fax: +81-27-2208215, E-mail: ishiget@gunma-u.ac.jp

Financial support: None. Conflict of interest: None. lescents with CD, as growth potential and pubertal development can be optimized only with effective disease control. ${ }^{2}$

Enteral nutrition (EN) was described as an effective treatment for CD in children by Peters in $1976 .{ }^{3}$ Exclusive enteral nutrition (EEN) refers to the exclusive use of an enteral formula (i.e., elemental formula, oligomeric formula, or a polymeric diet) as a distinct therapy; this reportedly has a positive effect on growth in patients with pediatric-onset CD. ${ }^{2,45}$ However, the use of and protocols for EN (exclusive vs. supplementary, type of formula, treatment duration, and indications for maintenance) vary among countries. ${ }^{6-9}$ Studies have reported that a polymeric diet is more frequently used as the formula type in Western countries, ${ }^{10}$ whereas an

\footnotetext{
(c) Copyright 2017. Korean Association for the Study of Intestinal Diseases. All rights reserved.

This is an Open Access article distributed under the terms of the Creative Commons Attribution Non-Commercial License (http://creativecommons.org/licenses/by-nc/4.0)

which permits unrestricted non-commercial use, distribution, and reproduction in any medium, provided the original work is properly cited.
} 
elemental formula is widely used in Japan. ${ }^{11}$ Treatment protocols for EEN with elemental formula for pediatric patients with CD have not been well documented. In the present study, we utilized a questionnaire to evaluate physicians' attitudes toward EN and assessed the EN treatment details in pediatric patients with CD in Japan.

\section{METHODS}

\section{Study Population}

All members of the working group for pediatric IBD treatment guidelines were contacted via e-mail and invited to participate in a survey in July 2012. The working group members included 32 of the 36 delegates to the Japanese Society of Pediatric IBD (23 pediatric gastroenterologists, 6 gastroenterologists, and 3 gastrointestinal surgeons). The majority of hospitals involved in the treatment of pediatric CD are members of this society. The survey participants were asked to complete and return the 2 questionnaires described below via e-mail by the end of August 2012. A second e-mail was sent after 4 weeks as a reminder to those who had not yet responded.

\section{Questionnaires}

Two types of questionnaires were developed for this study. The 16-item consensus questionnaire assessed the attitudes of physicians toward EN. The physicians were asked about their EN standard protocols, including whether they used EN as induction and maintenance therapy in pediatric patients with CD. They were also asked whether they believed that EN affected their patients' quality of life (QOL). If they indicated that in their opinion, EN worsened the patients' QOL, they were asked to report what interventions they recommended to minimize this effect.

The 14-item efficacy questionnaire collected data on patients aged $<17$ years who received induction therapy between 2006 and 2011. It assessed treatment details and patient demographics. Patients were diagnosed with CD based on standard criteria. ${ }^{12}$ To eliminate bias that could have resulted from differences in the number of patients treated in different facilities, physicians were asked to provide information regarding the last 5 patients they had treated, if they had treated $>5$ patients. Survey items included questions on patient profiles, number of relapses, types of treatments administered as induction therapy, formulas used for EN, EN duration, and whether remission was achieved without ad- ditional treatment (Table 1). Pediatric Crohn's Disease Activity Index (PCDAI) scores at the initiation of EN treatment, 2 weeks after treatment initiation, and at the end of treatment were also obtained. Differences in disease behavior and PCDAI scores at onset and 2 weeks after initiation of treatment between patients who did and those who did not achieve remission with EN were analyzed.

Remission was defined as an International Organization for the Study of Inflammatory Bowel Diseases score of 0 or 1 , and negative CRP, based on the Japanese guidelines for CD. EEN was defined as liquid enteral nutrient intake for all peroral or pernasal nutrition, irrespective of intravenous supplemental nutrition. Partial enteral nutrition (PEN) was defined as a combination of liquid EN and the "Crohn's diet" (low fat and low fiber diet). Regarding types of formula, the commercial product names of liquid EN products available in Japan (Elental ${ }^{\circledR}$ and Elental $\mathrm{P}^{\circledR}$, Ajinomoto Pharmaceuticals, Tokyo, Japan, for elemental formula; Twin-line $\mathrm{NF}^{\circledR}$, Otsuka Pharmaceutical, Tokyo, Japan; Peptino ${ }^{\circledR}$, Terumo, Tokyo, Japan, for oligomeric formula; Ensure Liquid ${ }^{\circledR}$, Abbott

Table 1. Elemental Nutrition Preferences of Surveyed Physicians

\begin{tabular}{lc}
\hline \multicolumn{1}{c}{ Question/option } & No. (\%) \\
\hline Newly diagnosed patients with CD & $18(82)$ \\
All patients should be treated with EN & 0 \\
EN should not be used & $4(18)$ \\
Depending on disease phenotype & \\
Patients with CD relapse & $13(59)$ \\
All patients should be treated with EN & $8(36)$ \\
Only use in cases in which EN was previously effective & $1(5)$ \\
EN should not be used & \\
As maintenance therapy & $14(64)$ \\
All patients should be treated with EN & $2(9)$ \\
EN should not be used & $6(27)$ \\
Depending on disease phenotype & \\
Type of EN recommended for patients with active CD & $18(82)$ \\
Exclusive EN & $1(5)$ \\
Partial EN & $2(9)$ \\
Depending on disease phenotype & $18(82)$ \\
Type of formula recommended for patients with CD & 0 \\
Elemental formula & \\
Oligomeric formula & \\
Delymeric diet & \\
\hline EN enteral nutrition. & \\
\hline
\end{tabular}


Japan, Tokyo, Japan; and Racol ${ }^{\circledR}$, Otsuka Pharmaceutical for polymeric diet) were listed in the questionnaire.

The questionnaire data included only de-identified, unlinkable, and anonymous data. The study design qualified for an exemption from the requirement for informed consent by the Gunma University Hospital Institutional Review Board.

\section{Statistical Analyses}

Assessment of the normality of the data was performed using SPSS version 22.0 (IBM Corp., Armonk, NY, USA). Fisher exact test was used to investigate the differences in disease behavior between patients who did and did not achieve remission with EN. The Wilcoxon signed-rank test was used to compare PCDAI scores before and after treatment with EN.

\section{RESULTS}

\section{Response Rate and Patient Demographics}

Of the 32 physicians we contacted, 23 (19 pediatric gastroenterologists, 2 gastroenterologists, and 2 surgeons) responded to the questionnaires.

Data from 58 patients treated in 15 hospitals were examined in the efficacy questionnaire. Only 1 case was reported from an adult gastroenterology department, whereas the others were reported by pediatric gastroenterologists. Five hospitals reported treating fewer than 5 cases. Of the 58 patients, $42(72 \%)$ were male patients. The age at diagnosis ranged from 0 to 16 years, with a median age of 12 years; the median patient age at the beginning of treatment was 13 years. A total of 44 patients (77\%) had newly diagnosed CD, whereas 14 patients were treated for a CD relapse.

\section{Consensus Questionnaire}

Of 22 physicians who responded to this questionnaire, 18 (82\%) recommended treatment with EN for all newly diagnosed pediatric patients with CD, whereas 4 (18\%) stated that they would consider administering EN only in certain situations, such as in patients with small intestinal involvement $(3 / 4)$, growth delay $(2 / 4)$, or in cases of adherence to EN by patients and parents (2/4) (Table 1$)$. In summary, all physicians recommended EN as a treatment option. In cases of CD relapse, 13 of the 22 physicians (59.1\%) recommended EN for all patients, whereas $8(36.4 \%)$ recommended it only for those who had responded to EN during the first induction therapy. Regarding maintenance therapy, EN was recommended for all patients by 14 of the 22 physicians (63.7\%); in contrast, 6 (27.2\%) recommended EN depending on patient phenotypes ( 2 severe cases, 2 patients with small intestinal involvement, 1 patient with a growth delay, and 1 patient based on age).

Of the 22 physicians, 18 (84\%) recommended EEN, as opposed to PEN, as induction therapy for all patients. Regarding the type of formula used for induction therapy, elemental formula was preferred by 18 of 21 of physicians (86\%), whereas a polymeric diet was preferred by 2 physicians (10\%). EEN duration was determined based on symptomatic improvement according to 16 of 22 physicians (73\%), whereas a limited treatment duration was used by 5 of 22 physicians (23\%). A polymeric diet was used as maintenance therapy by 10 of 23 physicians (43\%). The recommended caloric intake obtained from the enteral formula during maintenance therapy was approximately $30 \%$ of the total oral intake, according to the majority of the physicians.

When asked about the perceived effects of EN on their patients' QOL, 18 of 23 physicians (78\%) reported that the use of $\mathrm{EN}$ as induction therapy had a negative effect on their patients' QOL. Enhanced support by medical staff and family members was cited as the strategy most likely to improve the QOL of pediatric patients with CD as well as to increase their adherence to treatment with EN.

\section{Efficacy Questionnaire}

The disease phenotypes of all patients at the beginning of induction therapy are shown in Table 2. The majority of patients $(45 / 58,78 \%)$ had small intestinal involvement. A total of 4 of 58 patients (7\%) had strictures, although none had internal fistulas. PCDAI scores prior to treatment-available for 32 of the 58 patients-ranged between 10 and 77.5 (median score, 35). All patients except 1 received treatment with EN. Treatment details are shown in Table 3. Among the 57 patients treated with EN, 40 (70\%) and 17 (30\%) received EEN and PEN, respectively. Regarding formula types, the elemental formula Elental ${ }^{\circledR}$ was most commonly used, whereas a polymeric diet was used in only 2 patients (4\%). Among patients treated with EN (including EEN, PEN, and Crohn's diet), immunosuppressive treatments such as steroids or immunomodulators were simultaneously used in 17 patients (30\%). A total of 31 of 40 patients (80\%) treated with EN alone achieved remission without the requirement of any additional treatment other than 5-aminosalicylic acid, which was administered to 56 patients $(97 \%)$. Among the 40 patients who received EEN, 14 (35\%) simultaneously received 
Table 2. Patient Demographics (Efficacy Questionnaire)

\begin{tabular}{|c|c|}
\hline Demographics of patients enrolled & No. (\%) \\
\hline \multicolumn{2}{|l|}{ Age at disease onset (Paris classification) } \\
\hline A1a: 0-9 yr & $6(10)$ \\
\hline A1b:10-19 yr & $52(90)$ \\
\hline \multicolumn{2}{|l|}{ Sex } \\
\hline Male & $42(72)$ \\
\hline Female & $16(28)$ \\
\hline \multicolumn{2}{|l|}{ PCDAl score at disease onset } \\
\hline$<10$ & 0 \\
\hline $10-29$ & $12(38)$ \\
\hline $30-59$ & $17(53)$ \\
\hline$\geq 60$ & $3(9)$ \\
\hline NA & 26 \\
\hline \multicolumn{2}{|l|}{ Disease location (Paris classification) } \\
\hline L1: terminal ileal +/- limited cecal disease & $9(16)$ \\
\hline L2: colonic & $10(17)$ \\
\hline L3: ileocolonic & $28(48)$ \\
\hline L4: isolated upper disease & 0 \\
\hline$L 1+L 4 a$ & $1(2)$ \\
\hline$L 2+L 4 a$ & $3(5)$ \\
\hline$L 3+L 4 a$ & $7(12)$ \\
\hline \multicolumn{2}{|l|}{ Disease behavior (Paris classification) } \\
\hline B1: nonstricturing, nonpenetrating & $30(52)$ \\
\hline B1p: B1 with perianal disease & $24(41)$ \\
\hline B2: stricturing & $3(5)$ \\
\hline B2p: B2 with perianal disease & $1(2)$ \\
\hline \multicolumn{2}{|l|}{ Growth delay (Paris classification) } \\
\hline $\mathrm{G}_{1}$ : growth delay & $18(31)$ \\
\hline $\mathrm{G}_{0}$ & 40 (69) \\
\hline
\end{tabular}

PCDAl, Pediatric Crohn's Disease Activity Index; NA, not applicable.

immunosuppressive therapy. Of the remaining 26 patients, $22(85 \%)$ achieved remission without the need for additional therapy. The mean duration of EEN administration was 15.9 days. Of the 6 patients who received EEN for $>30$ days, 3 (50\%) did not achieve remission.

To evaluate whether PCDAI scores during the early phase (after 2 weeks) of EEN treatment could predict the efficacy of EEN, scores at the initiation of treatment and 2 weeks thereafter were analyzed for the 21 patients with available data. Among the 17 patients who responded to EEN, 9 had PCDAI scores $<10$ and 5 had scores of 10 to 20 at 2 weeks after the initiation of EN treatment. Only 1 of 4 patients who
Table 3. Active Treatment Types, Types of Enteral Nutrition, Formulas, and Response to Enteral Nutrition Therapy (Japan)

\begin{tabular}{|c|c|}
\hline Treatments received/outcome & No. $(\%)$ \\
\hline \multicolumn{2}{|l|}{ Active treatment types in patients with pediatric CD } \\
\hline EN & $57(98)$ \\
\hline 5-ASA & $56(97)$ \\
\hline Steroid & $23(40)$ \\
\hline Immunomodulator & $21(36)$ \\
\hline Biologics & $12(21)$ \\
\hline Antibiotics (metronidazole, ciprofloxacin) & $7(12)$ \\
\hline Granulocyte/monocyte apheresis & 0 \\
\hline Surgery & $2(3)$ \\
\hline \multicolumn{2}{|l|}{ Type of EN used } \\
\hline Exclusive EN & $40(70)$ \\
\hline Partial EN & $17(30)$ \\
\hline Crohn's diet & 0 \\
\hline EN not performed & $1(2)$ \\
\hline \multicolumn{2}{|l|}{$\begin{array}{l}\text { Type of formula used in patients treated with EN } \\
\text { (total/among EEN patient) }\end{array}$} \\
\hline Elental ${ }^{\circledR}$ (elemental formula) & $52(91) / 38(95)$ \\
\hline Elental $P^{\circledR}$ (elemental formula) & $1(2) / 1(3)$ \\
\hline Peptino ${ }^{\circledR}$ (oligomeric formula) & $2(4) / 1(3)$ \\
\hline Racol ${ }^{\circledR}$ (polymeric diet) & $2(4) / 0$ \\
\hline \multicolumn{2}{|l|}{ Response to EN (total/among EEN patient) } \\
\hline $\begin{array}{l}\text { Achieved remission without immunosuppressive } \\
\text { therapy }^{2}\end{array}$ & $31(79) / 22(85)$ \\
\hline Failed to achieve remission with EN alone & $8(21) / 4(15)$ \\
\hline
\end{tabular}

In response to enteral nutrition (EN) section, patients who received EN and immunosuppressive therapy simultaneously $(n=18)$ were excluded owing to inability to measure efficacy of EN treatment.

IImmunosuppressive therapy consisted of steroids, immunomodulators, biologics, and antibiotics.

5-ASA, 5-aminosalicylic acid; EEN, exclusive EN.

did not achieve remission with EEN showed a PCDAI score of $<20$. Thus, PCDAI scores 2 weeks after EEN initiation were significantly higher in nonresponders than in responders $(P<0.05$, Wilcoxon signed-rank test) (Fig. 1$)$.

\section{DISCUSSION}

EEN has been shown to be effective for the treatment of pediatric patients with $\mathrm{CD}$, and its use has been recommended in guidelines published by the European Crohn's and Colitis Organization (ECCO)/The European Society for Pediatric Gastroenterology, Hepatology, and Nutrition 


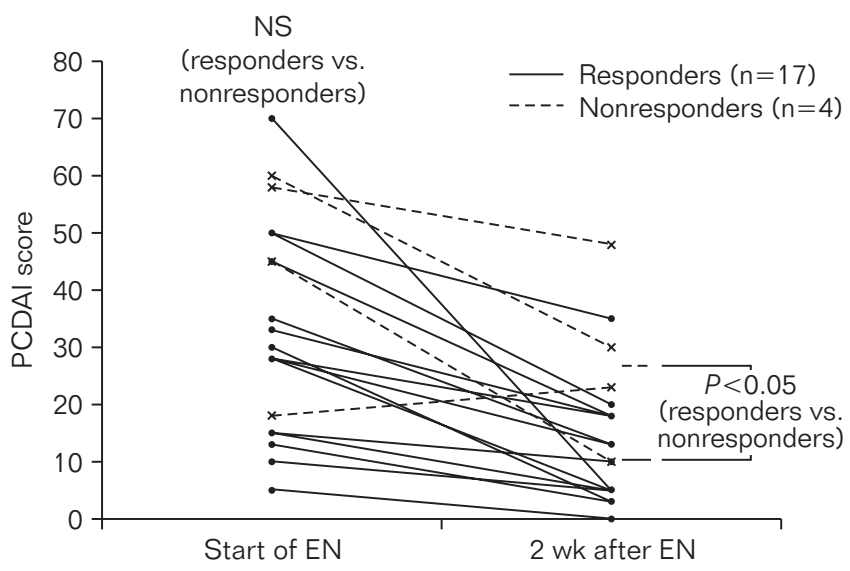

Fig. 1. Pediatric Crohn's Disease Activity Index (PCDAI) scores in patients treated with enteral nutrition (EN) alone. Paired data were available for 21 patients. Responders showed a marked and statistically significant $(P<0.05$, Wilcoxon signed-rank test) improvement in PCDAl scores after 2 weeks of treatment compared to nonresponders. A total of 16 of 17 responders and 1 of 4 nonresponders showed PCDAl scores $<20$ at 2 weeks after the start of treatment with EN.

(ESPGHAN), as well as in previous Japanese pediatric CD guidelines. ${ }^{13,14}$ EEN has also been recommended for use in adult patients with CD in Japan. ${ }^{15}$ However, a number of factors determine whether EEN is administered to pediatric patients. Reports have suggested that physician experience, concerns regarding treatment adherence, and high medical cost contribute to its limited use. Levine et al. ${ }^{8}$ showed that only $4 \%$ of pediatric gastroenterologists in North America employed EEN on a regular basis, compared to $62 \%$ of pediatric gastroenterologists in Western Europe. In Australia, Day et al. ${ }^{6}$ reported that 6 of 21 doctors regularly used EEN, 6 occasionally used EEN, and 9 did not use EEN or used it rarely; the authors suggested that experience with EEN during training was positively associated with the current use of EEN.

In the present study, we found that EEN was widely used by pediatric gastroenterologists in Japan. The majority $(18 / 22,82 \%)$ of surveyed physicians regularly prescribed EEN for pediatric patients newly diagnosed with CD. Furthermore, EEN was not only used as induction therapy for patients with new-onset $\mathrm{CD}$, but also in cases of relapse. Although ECCO/ESPGHAN guidelines suggest that the efficacy of EEN for patients with severe pancolitis and perianal lesions is unclear, ${ }^{13}$ we found that the majority of physicians surveyed in the present study selected EN regardless of the patients' disease phenotypes.

Regarding the types of formula used for EN, only a few studies have suggested that elemental formula is superior to a polymeric diet as induction therapy in patients experienc- ing CD remission; ${ }^{16}$ nevertheless, the physicians surveyed in the present study reported commonly using elemental formula. Elemental formula and some types of polymeric diets are covered by insurance and financial aid for intractable diseases in Japan; hence, elemental formula is commonly used in adult patients. Furthermore, elemental formula has been reported to be effective as both induction and maintenance therapy in adult Japanese patients with $\mathrm{CD} .{ }^{17}$ Our findings suggest that physicians treating pediatric $\mathrm{CD}$ also commonly use elemental formula in Japan. The majority of surveyed physicians $(17 / 22,76 \%)$ reported that treatment with EEN was continued until the patients' symptoms improved. Data derived from the efficacy questionnaire indicated that most patients received EEN for only 2 weeks (thus, short-term); this period is significantly shorter than the EEN treatment duration of 6 to 8 weeks in Western countries. ${ }^{10}$

The efficacy questionnaire revealed that 22 of the 26 patients $(85 \%)$ who began therapy with EEN alone achieved remission without additional treatments. This finding is similar to data reported in Western countries, where EEN is typically administered along with a polymeric diet for 6 to 8 weeks. ${ }^{10}$ In a prospective study, Grogan et al. ${ }^{18}$ reported that $93 \%$ and $79 \%$ of patients who received elemental formula and a polymeric diet, respectively, achieved remission after 6 weeks of EEN treatment. Furthermore, de Bie et al. ${ }^{19}$ reported that EEN treatment with hyperosmolar sip feeds or a polymeric diet for 6 weeks resulted in complete remission in 55 of 77 patients (71\%); however, EEN was discontinued in 10 of 77 patients (13\%) due to nonadherence. The findings of the present study indicate similar efficacy with a shorter EEN treatment duration. A shorter treatment duration would likely improve the QOL of patients, as well as their adherence to therapy. In contrast, only 3 of 6 patients who received EEN for 30 days responded to treatment in this study. The significant decrease in PCDAI scores 2 weeks after the start of EEN treatment suggests that EEN is effective after a short time. If PCDAI scores do not improve to a score of 20 or less within 2 weeks of treatment, physicians might prefer switching from EEN to other therapies.

The present study has several limitations. This study only included 23 physicians. Moreover, only $68 \%$ of the physicians in the present study completed the questionnaires. However, the Japanese Society of Pediatric IBD consists of 36 chair members. The majority of hospitals treating multiple pediatric CD cases in Japan are thought to have physicians who have joined the society as chair members. All of the physicians in the present study were among these chair members. Thus, the majority of Japanese physicians treating 
pediatric patients with CD were likely included in this survey. Not all survey participants reported data on 5 patients in the efficacy questionnaire, which could have resulted in selection bias. Moreover, 13 of 40 patients were treated with EEN and immunosuppressive therapy simultaneously. These patients might have had more severe disease phenotypes than patients who received EEN alone, resulting in inaccurate EEN remission rates. Furthermore, outcome measures other than PCDAI scores, such as nutritional status, growth improvement, or inflammatory status, were not assessed in this study.

Although the use of EEN with an elemental formula with a rather short treatment duration demonstrated remission rates that were comparable to those reported in Western countries, it is unclear whether this treatment approach is as effective as EEN with a polymeric diet over 6 to 8 weeks (as preferred in Western countries), since we have no data involving placebo or polymeric diet treatment. An EEN treatment duration of only 2 weeks might markedly improve the QOL of patients. Hence, we suggest that a future study should evaluate whether elemental formula is superior to a polymeric diet over a 2-week period.

In conclusion, we found that EEN was widely recommended by pediatric gastroenterologists in Japan. Despite the lack of clear evidence, it appears that elemental formula was preferred over a polymeric diet, and that supplemental elemental formula is commonly used in Japan. Our data indicated a rather short EEN duration of 2 to 3 weeks in the majority of pediatric patients with CD. Further study is required to assess whether elemental formula is superior to a polymeric diet over a 2-week treatment period.

\section{ACKNOWLEDGEMENTS}

We wish to thank Katsuhiro Arai, Daiki Abukawa, Itaru Iwama, Yoshikazu Otsuka, Reiko Kunisaki, Mitsuru Kubota, Hiroyuki Yamada, Seiichi Kagimoto, Mutsuko Konno, Nagamu Inoue, Shigeru Toyoda, Riichiro Nezu, Shun-ichi Maisawa, Takahiro Mochizuki, Kosuke Ushijima, Akira Andoh, Satoru Nagata, Keiichi Uchida, and Tomoki Aomatsu for their assistance and the provision of relevant data. We also acknowledge Hirokazu Arakawa, Professor in the Department of Pediatrics, Gunma University Graduate School of Medicine, for valuable help with data analysis and English revision.

\section{REFERENCES}

1. Ishige T, Tomomasa T, Takebayashi $\mathrm{T}$, et al. Inflammatory bowel disease in children: epidemiological analysis of the nationwide IBD registry in Japan. J Gastroenterol 2010;45:911-917.

2. Heuschkel R, Salvestrini C, Beattie RM, Hildebrand H, Walters T, Griffiths A. Guidelines for the management of growth failure in childhood inflammatory bowel disease. Inflamm Bowel Dis 2008;14:839-849.

3. Peters H. Parenteral-peroral combined treatement of Crohn's disease and ulcerative colitis. Infusionsther Klin Ernahr 1976;3:222-226.

4. Day AS, Whitten KE, Lemberg DA, et al. Exclusive enteral feeding as primary therapy for Crohn's disease in Australian children and adolescents: a feasible and effective approach. J Gastroenterol Hepatol 2006;21:1609-1614.

5. Shamir R, Phillip M, Levine A. Growth retardation in pediatric Crohn's disease: pathogenesis and interventions. Inflamm Bowel Dis 2007;13:620-628.

6. Day AS, Stephenson T, Stewart M, Otley AR. Exclusive enteral nutrition for children with Crohn's disease: use in Australia and attitudes of Australian paediatric gastroenterologists. J Paediatr Child Health 2009;45:337-341.

7. Stewart M, Day AS, Otley A. Physician attitudes and practices of enteral nutrition as primary treatment of paediatric Crohn disease in North America. J Pediatr Gastroenterol Nutr 201 1;52:3842.

8. Levine A, Milo T, Buller H, Markowitz J. Consensus and controversy in the management of pediatric Crohn disease: an international survey. J Pediatr Gastroenterol Nutr 2003;36:464-469.

9. Whitten KE, Rogers P, Ooi CY, Day AS. International survey of enteral nutrition protocols used in children with Crohn's disease. J Dig Dis 2012;13:107-112.

10. Day AS, Whitten KE, Sidler M, Lemberg DA. Systematic review: nutritional therapy in paediatric Crohn's disease. Aliment Pharmacol Ther 2008;27:293-307.

11. Yamamoto T, Nakahigashi M, Saniabadi AR. Review article. Diet and inflammatory bowel disease: epidemiology and treatment. Aliment Pharmacol Ther 2009;30:99-112.

12. Yao T, Matsui T, Hiwatashi N. Crohn's disease in Japan: diagnostic criteria and epidemiology. Dis Colon Rectum 2000;43(10 Suppl):S85-S93.

13. Ruemmele FM, Veres G, Kolho KL, et al. Consensus guidelines of ECCO/ESPGHAN on the medical management of pediatric Crohn's disease. J Crohns Colitis 2014;8:1179-1207. 
14. Working Group of the Japanese Society for Pediatric Gastroenterology, Hepatology and Nutrition, Konno M, Kobayashi A, et al. Guidelines for the treatment of Crohn's disease in children. Pediatr Int 2006;48:349-352.

15. Ueno F, Matsui T, Matsumoto T, et al. Evidence-based clinical practice guidelines for Crohn's disease, integrated with formal consensus of experts in Japan. J Gastroenterol 2013;48:31-72.

16. Giaffer MH, North G, Holdsworth CD. Controlled trial of polymeric versus elemental diet in treatment of active Crohn's disease. Lancet 1990;335:816-819.
17. Matsui T, Sakurai T, Yao T. Nutritional therapy for Crohn's disease in Japan. J Gastroenterol 2005;40 Suppl 16:25-31.

18. Grogan JL, Casson DH, Terry A, Burdge GC, El-Matary W, Dalzell AM. Enteral feeding therapy for newly diagnosed pediatric Crohn's disease: a double-blind randomized controlled trial with two years follow-up. Inflamm Bowel Dis 2012;18:246-253.

19. de Bie C, Kindermann A, Escher J. Use of exclusive enteral nutrition in paediatric Crohn's disease in the Netherlands. J Crohns Colitis 2013;7:263-270. 